\title{
Erratum to: Parametric Response Mapping of Longitudinal PET Scans and Their Use in Detecting Changes in Alzheimer's Diseases
}

Seung-hak Lee and Hyunjin Park, the ADNI ${ }^{\dagger}$

(C) The Korean Society of Medical \& Biological Engineering and Springer 2014

In the volume 4, issue 1 of the Biomedical Engineering Letters, an error occurred in the research article:

Seung-hak Lee and Hyunjin Park, the ADNI ${ }^{\dagger}$

Parametric Response Mapping of Longitudinal PET Scans and Their Use in Detecting Changes in Alzheimer's Diseases

Biomed Eng Lett. (2014) 4:68-76 DOI 10.1007/s13534-0140120-8
- In the page number

Original wording:

Biomed Eng Lett (2014) 4:86-92

This should be replaced by:

Biomed Eng Lett (2014) 4:73-79

Seung-hak Lee, Hyunjin Park $(\square)$

School of Electronic and Electrical Engineering, Sungkyunkwan University, Suwon 440-746, Korea

Tel : +82-31-299-4956 / Fax : +82-31-290-5819

E-mail : hyunjinp@skku.edu

'Data used this article were obtained from the Alzheimer's Disease Neuroimaging Initiative (ADNI) database. 